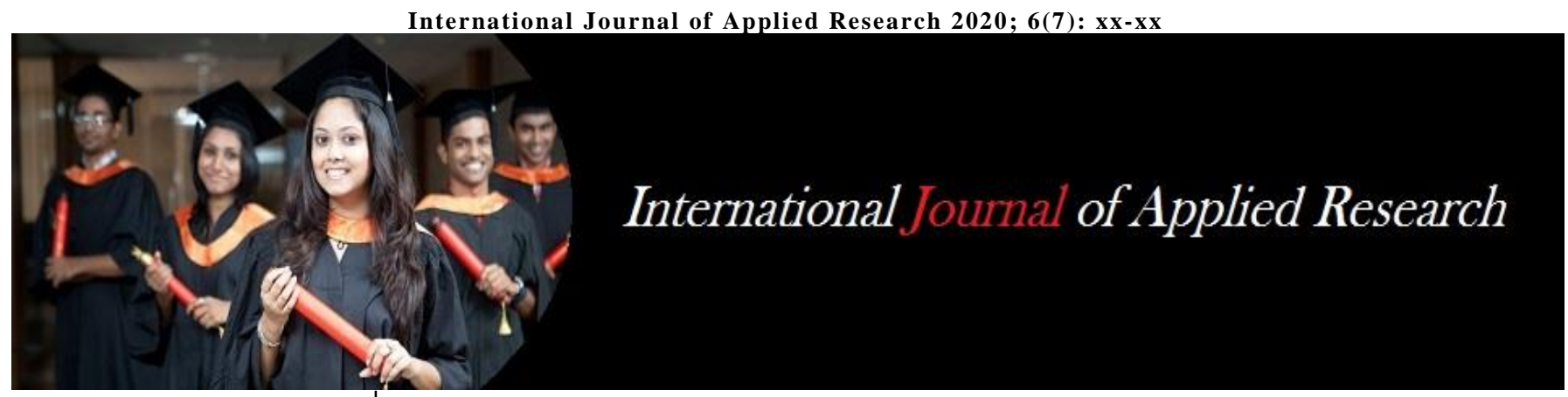

ISSN Print: 2394-7500 ISSN Online: 2394-5869 Impact Factor: 5.2 IJAR 2020; 6(7): xx-xx www.allresearchjournal.com Received: 19-06-2020 Accepted: 21-07-2020

Dr. Siddhima Hardikar Assistant Professor

(Neuroscience), Modern Colleges of Physiotherapy,

Pune, Maharashtra, India

Dr. Sanjiv Kumar Principal, KLE Institute of Physiotherapy, Belgavi, Karnataka, India
Corresponding Author: Dr. Siddhima Hardikar Assistant Professor (Neuroscience), Modern Colleges of Physiotherapy, Pune, Maharashtra, India

\section{Comparative study of Faradic muscle stimulation with myofascial release and Faradic muscle stimulation with passive stretching to improve hand muscle function in chronic stroke patients: A randomized controlled trial}

\section{Dr. Siddhima Hardikar and Dr. Sanjiv Kumar}

DOI: https://doi.org/10.22271/allresearch.2020.v6.18c.6999

\section{Abstract}

Objective: To compare effectiveness of Faradic muscle stimulation with myofascial release and Faradic muscle stimulation with passive stretching to improve hand muscle function in chronic stroke patients as compared to conventional exercise protocol.

Background: There is limited study available that compares myofascial release and passive stretching, with the conventional exercise protocol used for treatment to improve hand muscle function in chronic stroke patients.

Study design: Randomized controlled trial

Methods: Ethical clearance was taken from college and consent was taken from the 30 subjects diagnosed with stroke and divided into 3 equal group by chit method. Pre and post outcome measure of MSHFT (Modified Sollerman Hand Function Test) was taken. Group A underwent Faradic Muscle Stimulation with myofascial release, group B underwent Faradic Muscle Stimulation with passive stretching and group C underwent Faradic Muscle Stimulation with home exercise programme. Faradic electrical muscle stimulation was given for wrist extensors.

Results: Mean values for MSHFT for Group A was: $4.2 \pm 0.15$; Group B was: $3.8 \pm 0.55$; Group C was: $2.2 \pm 0.43$

Conclusion: In the present study it was found that Faradic muscle stimulation along with myofascial release and Faradic muscle stimulation along with passive stretching are more effective than conventional exercise protocol in management of stroke.

Keywords: Stroke, Faradic muscle stimulation, myofascial release, MSHFT, passive stretching

\section{Introduction}

- Stroke was first described as the phenomenon of sudden paralysis by Hippocrates. According to WHO in 1970, stroke is characterized by rapidly developed clinical signs of focal disturbance of cerebral function of presumed vascular origin and of more than 24 hours duration. A stroke, also known as a cerebrovascular accident (CVA), is the rapid loss of brain function due to disturbance in the blood supply to the brain.

- This can be due to ischemia (lack of blood flow) caused by blockage (thrombosis, arterial embolism), or a haemorrhage (leakage of blood) ${ }^{[1]}$. As a result, the affected area of the brain cannot function, which might result in an inability to move one or more limbs on one side of the body, inability to understand or formulate speech, or an inability to see one side of the visual field ${ }^{[2]}$.

- Because of loss of blood supply to the brain, brain tissue ceases to function if deprived of oxygen for more 60 to 90 seconds and approximately after 3 hours, will suffer irreversible injury leading to death of tissue ${ }^{[3]}$. Since the oxygen or glucose becomes depleted in ischemic brain tissue, the production of high energy phosphate compound such as adenosine triphosphate fails, leading to failures of energy dependent processes, necessary for tissue cell survival ${ }^{[4]}$.

- Stroke is diagnosed through several techniques: a neurological examination, CT scans (most often without contrast enhancements) or MRI scans, Doppler ultrasound, and 
arteriography. The diagnosis of stroke itself is clinical, with assistance from the imaging techniques ${ }^{[5]}$.

- Treatment of spasticity related to stroke often involves early mobilizations, combined with elongation of spastic muscles and sustained stretching through various positioning.

- Electrical stimulation to the antagonist muscles or vibrations has been used with some success ${ }^{[6]}$.

- Conventional rehabilitation programs include methods such as exercise training of the affected hand, functional electrical stimulation, robotic-assisted rehab, impairment-oriented training of the arm, and bilateral (both) arm training ${ }^{[7]}$.

- Up to 85 percent of stroke patients experience paralysis of one side of the body. One-half to three-fourths of survivors have continued limitation in an upper extremity. While restoring voluntary control of skilled reaching and grasping movements is often a primary goal, $60 \%$ of stroke survivors continue to have significant upper limb disability after 6 months. However more recent evidences from studies of neuroplasticity suggest that changes in motor behaviour continuously occur across the lifespan with task practice or under different motor learning conditions. Theoretically neuroplasticity concept would suggest that some improvement of all functional movements may be possible after stroke, regardless of severity, if extensive learning and consistent practice occcurs ${ }^{[8]}$.

- Stroke survivors often experience chronic motor deficits, especially in the hand, resulting in diminished capacity to manipulate objects with the hand affected by the stroke. These deficits may not relate only to proper creation of grasp posture (hand opening and closing) but also to the timely release of the objects being held in the typical upper extremity posture (i.e. flexed elbow, pronated forearm, flexed wrist)

- Thus the observed difficulties in letting go of objects may result from sustained finger flexor muscle activity or inability to terminate finger flexor muscle in timely manner, delays in grip force scaling during grip-and-lift tasks, deficits in timing and coordination of movement and thus decreased motor firing.

- There are many documented physiotherapeutic treatments for hand function in chronic stroke, such as electrical stimulation, splinting, massage, passive movements. Electrical stimulation has been proven to improve biomechanical and functional movement parameter significantly ${ }^{[9]}$.

- Faradic muscle stimulation with restricted hand function in chronic stroke patients showed improved awareness of affected arm, hence it significantly improved hand muscle function and thus reducing spasticity ${ }^{[10]}$.

- In hemiplegia, patient undergoes severe structural tightness of ligaments, fascia, muscle, and joint capsule. It is due to musculoskeletal asymmetries that occur from abnormal tone, imbalances of muscle recruitment, prolonged static posturing, impaired mobility, and movement in repetitive abnormal patterns. The lack of motor functions contributes to asymmetries with development of fascial restrictions contributing to compensations further restricting movement and functional return ${ }^{[11]}$.
- Myofascial release approach is a form of soft tissue therapy used to treat somatic dysfunction and resulting pain and restriction of motion. It is a treatment described by Andrew Taylor Still, founder of osteopathy/osteopathic medicine, which uses continual palpatory feedback to achieve release of myofascial tissues. ${ }^{12}$ This is supposedly accomplished by relaxing contracted muscles, increasing circulation and lymphatic drainage, and stimulating the stretch reflex of muscles and overlying fascia ${ }^{[13]}$.

- The gentle traction applied to the restricted fascia will result in heat and increased blood flow in the area. This allows the body's inherent ability for self-correction to return, thus eliminating pain and restoring the optimum performance of the body. This concept was suggested by Paul Svacina and he developed principals for indirect myofascial release. Fascia covers all organs of the body, Myofascial stretching in one area of the body can be felt in and will affect the other body areas. Release of myofascial restrictions can affect other body organs through a release of tension in the whole fascia system. Myofascial release techniques work even though the exact mechanism is not yet fully understood.

- Along with myofascial release or as an independent treatment program in patients with stroke, passive stretching is used to decrease tightness and hence reduce spasticity. Passive stretching is a form of in which an external force exerts upon the limb to move it into the new position. This contrasts with active stretching. Passive stretching resistance is normally achieved through the force of gravity on the limb or on the body weighing down on it. It can also be achieved with the help of a partner, stretch bands, or mechanical devices. The more force exerted on the limb, the less time it will take to drop lower into the stretch. It is a fallacy that passive stretching will lead to 'an increase in injury risk'. Slow, relaxed stretching is useful in relieving spasms in muscles that are healing after an injury ${ }^{[14]}$.

\section{Need of Study}

- Stroke is the third most common cause of mortality after heart disease and cancer in India. Prevalence rates of stroke vary from one study to another, and there has been a definite increase in the prevalence and incidence of stroke disorder in India over the last 30 years.

- Stroke survivors often experience chronic motor deficits, especially in the hand, resulting in diminished capacity to manipulate objects with the hand affected by the stroke.

- There are a few studies available for measuring the effectiveness of myofascial release to reduce spasticity in muscles of stroke patients

- There are a few studies available for measuring the effectiveness of passive stretching to reduce spasticity in muscles of stroke patients

- There is limited study available that compares myofascial release and passive stretching, with the conventional exercise protocol used for treatment to improve hand muscle function in chronic stroke patients.

- Hence present study has been conducted.

\section{Aim}


- To compare effectiveness of Faradic muscle stimulation with myofascial release and Faradic muscle stimulation with passive stretching to improve hand muscle function in chronic stroke patients as compared to conventional exercise protocol.

\section{Objectives}

i. To study the effectiveness of myofascial release in hand muscle function in chronic stroke patients.

ii. To study the effectiveness of passive stretching in hand muscle function in chronic stroke patients.

iii. To study the effectiveness of faradic electrical muscle stimulation and home exercise program in hand muscle function in chronic stroke patients.

iv. To compare the effectiveness of faradic electrical muscle stimulation with myofascial release and faradic electrical muscle stimulation with passive stretching in hand muscle function in chronic stroke patients.

\section{Hypothesis}

Null hypotheses $\left(\mathbf{H}_{0}\right)$ : There is no effect of myofascial release, faradic muscle stimulation, passive stretching in chronic stroke patients.

Alternate hypotheses $\left(\mathbf{H}_{\mathbf{a}}\right)$ : There is effect of myofascial release, faradic muscle stimulation, passive stretching in chronic stroke patients.

\section{Materials}

Pen

Record or data collection sheet

Key

Coins

Couch/plinth and pillow

Faradic electrical muscle stimulator: Technomed Electronics-Microstim genius S.I NO T-1202-T 1203

Modified Sollerman Hand Function Test

\section{Methodology}

- Study design: Randomized control trial

- Sample size: 30

- Sampling technique: convenient sampling

- Allocation method: random allocation with chit method

- Study population: Both males and females between the ages 35 to 75 within a year of recovery from the first stroke

- Study settings:

KLE'S Prabhakar Kore Charitable Hospital and medical research center, Belgaum
KLE'S Kankanwadi Ayurvedic hospital, Belgaum

- Duration of data collection: 12 months

- Study intervention: 6 days per week for 2 weeks

\section{Inclusion Criteria}

- Both males and females between ages 35 to 75 years

- Subjects should have first ever stroke

- Subjects should have at least 100 passive wrist extension

- Subjects should have at least $90 \%$ of passive extension of fingers and thumb with wrist in neutral position

- Subjects should be medically stable

\section{Exclusion criteria}

- Mentally unstable subjects

- Subjects with cardiac pacemakers

- Subjects with uncontrolled cardiac arrhythmias

- Other central/peripheral neurological diseases other than stroke

- Subjects with altered sensorium

\section{Procedure}

- Ethical clearance was taken from the ethical committee of the college.

- Subjects were selected according to the inclusion and exclusion criteria from the Hospitals in and around the city.

- Subjects were explained about the study.

- Consent was taken from the patients who wished to participate in the study.

- Subjects were divided into 3 groups of 10 each: Group A, Group B and Group C by random allocation chit method.

- Subjects were assessed pre-treatment and modified sollerman hand function test (MSHFT) scores were recorded.

- Subjects in Group A were given Faradic muscle stimulation for wrist extensors along with myofascial release for flexor compartment of forearm and hand

- Subjects in Group B were given Faradic muscle stimulation for wrist extensors along with passive stretching of forearm and wrist flexors

- Subjects in Group C were given Faradic muscle stimulation for wrist extensors along with home exercise programme in form of self-stretching of wrist and forearm flexors and active exercises of hand

- Post treatment MSHFT scores were recorded.

Table

\begin{tabular}{|c|c|c|}
\hline Group A & Group B & Group C \\
\hline $\begin{array}{c}\text { Faradic muscle stimulation for wrist extensors once a day } \\
\text { for 2 weeks }\end{array}$ & $\begin{array}{c}\text { Faradic muscle stimulation } \\
\text { for wrist extensors once a day } \\
\text { for 2 weeks }\end{array}$ & $\begin{array}{c}\text { Faradic muscle stimulation for wrist } \\
\text { extensors once a day for 2 weeks }\end{array}$ \\
\hline $\begin{array}{c}\text { Myofascial release for flexor compartment of forearm and } \\
\text { hand was performed over 10 sessions of decreasing } \\
\text { frequency over 2 weeks. 6 sessions in } 1^{\text {st }} \text { week and } 4 \\
\text { sessions in } 2^{\text {nd }} \text { week. }\end{array}$ & $\begin{array}{c}\text { Passive stretching of forearm } \\
\text { and wrist flexors performed } \\
\text { once a day for 2 weeks. }\end{array}$ & $\begin{array}{c}\text { Home exercise programme in form of self- } \\
\text { stretching of wrist and forearm flexors and } \\
\text { active exercises of hand done once a day for } \\
2 \text { weeks. }\end{array}$ \\
\hline
\end{tabular}

\section{Interventions}

\section{$>$ Myofascial Release Technique:}

The subject is placed in sitting or supine position entire forearm from elbow to fingers carefully exposed. The area is cleaned with cotton. The upper limb positioned in mid flexion and supination. MFR is given for flexor compartment of forearm and hand.

\section{- For Biceps Brachii:}


The treatment procedure included, a focused stretch of the biceps brachii using the thumb of one hand and several tightly adducted fingers of the other hand. A vertical gross stretch of biceps brachii using one hand. Broad surface of the thumb is placed on one side of the muscle belly and several tightly adducted fingers on the other side, it is being held and when some amount of release or relaxation is felt it is stretched again by increasing vertical lift. It is been repeated until an end-feel is reached.

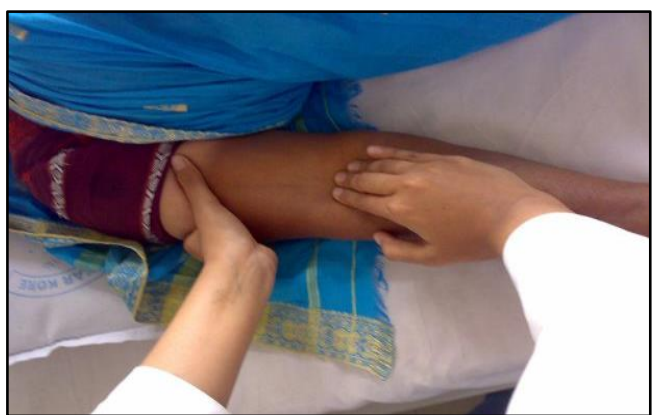

Image 1: Focused stretch for biceps

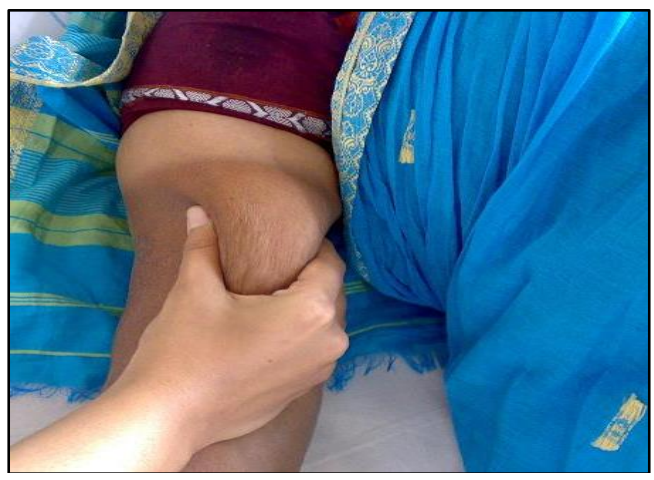

Image 2: Vertical stretch for biceps

\section{- For Brachialis}

A gross motor stretch of brachialis is performed with two or more slightly adducted fingers of each hand on the exposed lateral borders of this flat muscle that lies under the belly and tendon of the biceps brachii, it is stretched down towards the patients hand with firm gentle pressure.

\section{- For Brachioradialis}

Vertical gross stretch of brachioradialis using the broad surface of the thumb on one side of the muscle belly and several tightly adducted fingers. Vertical focused stretch is performed of the brachioradialis using several tightly adducted fingers to apply the stretch.

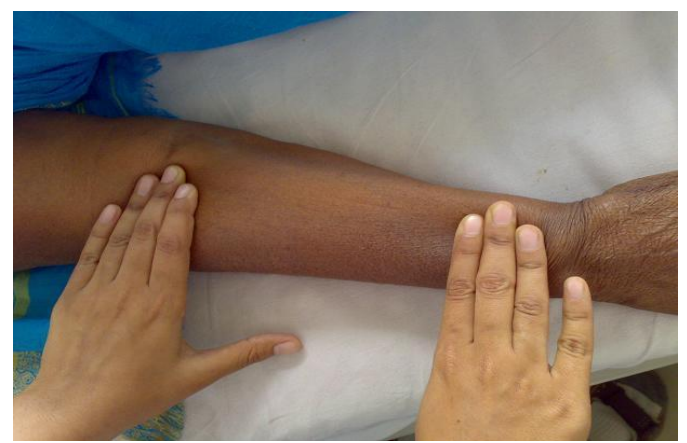

Image 3: Focussed stretch for forearm flexors

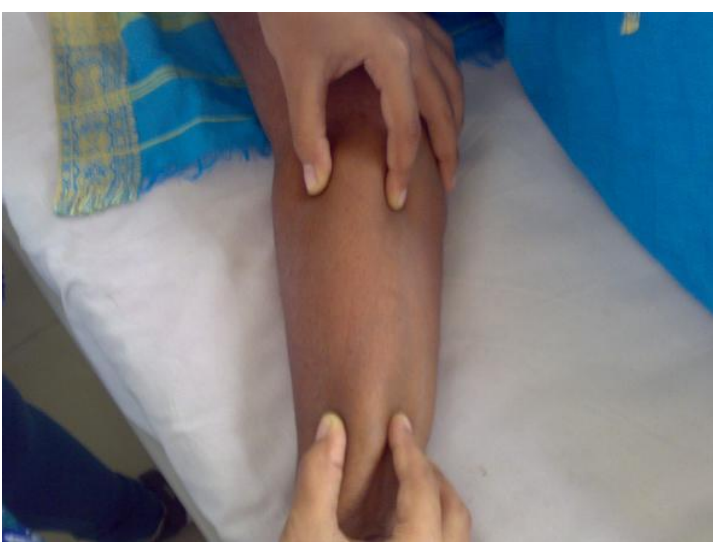

Image 4: Gross stretch for forearm flexors

\section{- For Pronater Teres}

A gross stretch of pronater teres using both the thumbs. A focused stretch of the flexors by gripping the muscle bellies between the thumb and one finger of both hands. Each flexor may be stretched individually by modifying this hand placement in response to feedback.

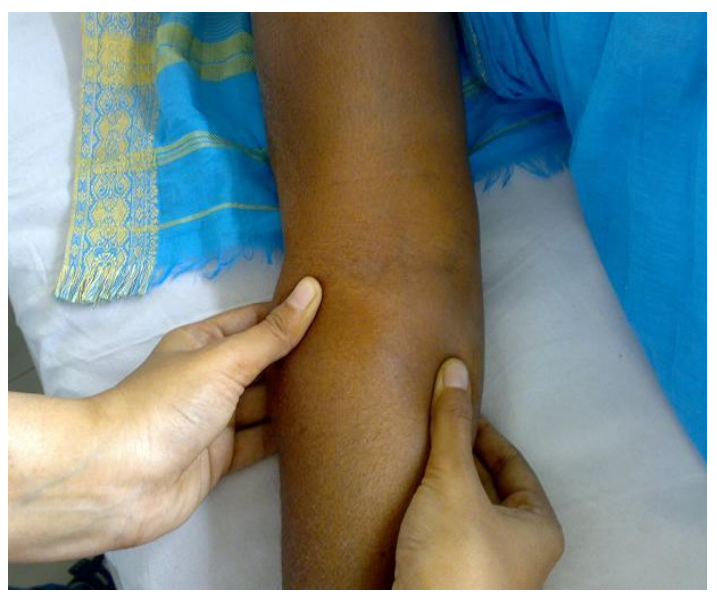

Image 5: Focussed stretch for pronater teres

\section{- For Flexor Carpi Radialis}

A focused stretch of flexor carpi radialis using both thumbs on the muscle belly or the thumb of one hand and several tightly adducted fingers of the other hand

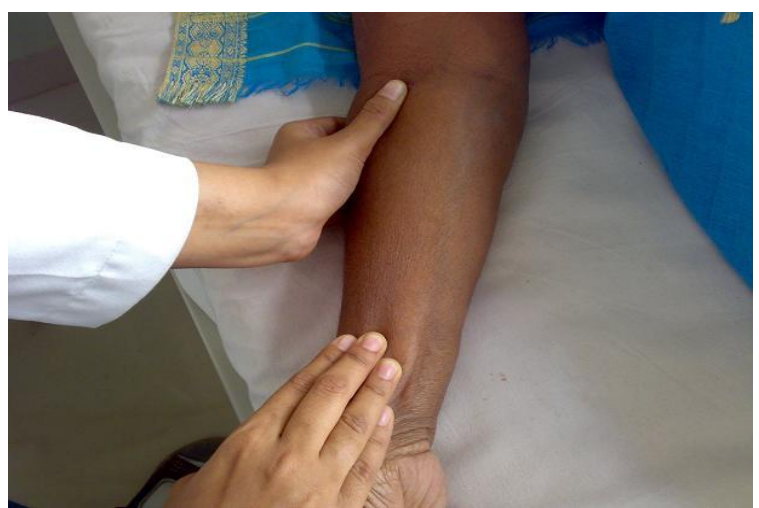

Image 6: Focussed stretch for flexor carpi radialis

- For Flexor Carpi Ulnaris

A focused stretch of flexor carpi ulnaris using both the thumbs on the muscle belly or the thumb of one hand and several tightly adducted fingers of the other hand. 


\section{- For Adductor Pollicis}

A focused stretch of adductor pollicis using one finger of each hand or both thumbs, wait for the release and stretched again, when an end feel is reached, the thumb is moved upward, and transverse head is stretched proximal to metacarpal.

\section{- For Flexor Pollicis Brevis}

A focused stretch of flexor pollicis brevis is performed using one finger of each hand or both thumbs, after release it is stretched again, and when an end feel is reached ,thumb is moved towards the wrist to stretch adductor pollicis brevis and indirectly stretch the opponens pollicis.

\section{- For interosseous}

A focused stretch of an interosseous using both thumbs are performed.

\section{- For Abductor digiti minimi}

A gross stretch of abductor digiti minimi using the thumb of one hand and one finger of other hand. Gently each finger is stretched so that all the soft tissue attachments around the joints and to decompress the joints, it is being held till the release and stretched again. the stretch sequence is repeated on each finger and thumb until the end -feel is reached.

Each subject is treated with MFR for 10 sessions of decreasing frequency over 2 -week period $.1^{\text {st }}$ week -6 sessions, 2nd week-4 sessions. After this intervention subjects were given faradic electrical muscle stimulation. Stimulation intensity adjusted according to subject's tolerance at the beginning and was readjusted if necessary. Interventions performed once a day for 2 weeks. Outcome measure is assessed post 2-week treatment by Modified Sollerman Hand Function Test to measure grip.

\section{Passive stretching}

Therapeutic interventions by faradic electrical muscle stimulation are carried out as explained in group A. After this intervention subjects were given passive muscle stretching for 30 minutes. Impaired forearm and hand is placed in supine position, the affected limb is taken passively into shoulder extension elbow extension and wrist and finger extension and a prolonged stretch is maintained until some relaxation is felt. At each session passive stretch is applied to wrist and forearm flexors in such a way that to produce considerable amount of relaxation for total 30 minutes. Intervention performed once a day for 2 weeks

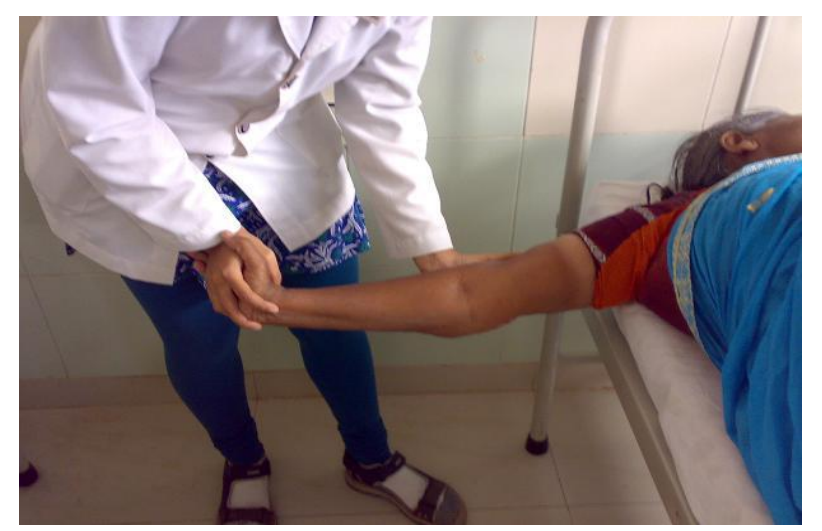

Image 7: Passive stretching of forearm and wrist flexors

\section{$>$ Faradic muscle stimulation}

The subject is placed in sitting or supine position entire forearm from elbow to fingers carefully exposed. Impaired forearm and hand are placed in prone position. At each session faradic electrical muscle stimulation is applied to wrist extensors in such a way to produce wrist extension contraction with 20 seconds rest in between the contractions for total 30 minutes. Stimulation intensity adjusted according to subject's tolerance at the beginning and was readjusted if necessary. Interventions performed once a day for 2 weeks.

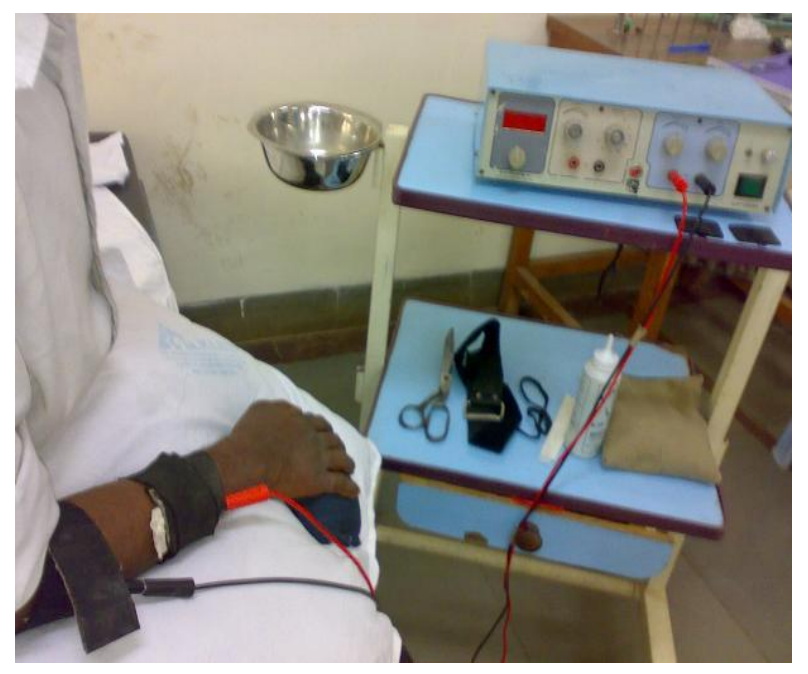

Image 8: Faradic muscle stimulation for wrist extensors

\section{Outcome Measure}

Modified Sollerman Hand Function Test (MSHFT)

Reliability and validity of MSHFT

Reliability $=0.96$ to 0.98

Validity $=<0.05$

\section{Review of Literature}

- Stott, D. J. (1999) et al. ${ }^{[15]}$ It has been suggested that electrical stimulation (ES) may enhance motor recovery after stroke. 60 hemiparetic patients (mean age, 68 years) 2 to 4 weeks after stroke into a randomized, controlled, parallel-group study comparing standard rehabilitation treatment with standard treatment plus ES of wrist extensors (3 times 30 minutes daily for 8 weeks). Isometric strength of wrist extensors was measured using a device built for that purpose. Upperlimb disability was assessed with use of the Action Research Arm Test (ARAT). Observations were continued for 32 weeks ( 24 weeks after the finish of ES or the control intervention phase). ES of the wrist extensors enhances the recovery of isometric wrist extensor strength in hemiparetic stroke patients. Upperlimb disability was reduced after 8 weeks of ES therapy, with benefits most apparent in those with some residual motor function at the wrist.

- Amir H Bakhtiary et al 2008. ${ }^{[16]}$ Forty stroke patients (aged from 42 to 65 years) with ankle plantar flexor spasticity were taken. Fifteen minutes of inhibitory Bobath techniques were applied to one experimental group and a combination of 9 minutes of electrical stimulation on the dorsiflexor muscles and inhibitory Bobath techniques was applied to another group for 20 sessions daily. The study concluded Therapy combining Bobath inhibitory technique and electrical stimulation 
may help to reduce spasticity effectively in stroke patients.

- Kuen-Horng Tsai Et al 2001. [17] The control of spasticity is often a significant problem in the management of patients with spasticity. The aim of this study was to evaluate the effect of a single session of prolonged muscle stretch (PMS) on the spastic muscle. Seventeen patients with spastic hemiplegia were selected to receive treatment. Subjects underwent PMS of the triceps surae (TS) by standing with the feet dorsiflexed on a tilt-table for 30 minutes. The test battery consisted of four measurements including the modified Ashworth scale of the TS, the passive range of motion (ROM) of ankle dorsiflexion, the H/M ratio of the TS, and the F/M ratio of the tibialis anterior (TA). The results indicated that the passive ROM of ankle dorsiflexion increased significantly $(\mathrm{p}<0.05)$ compared to that before PMS treatment. Additionally, PMS reduced motor neuron excitability of the TS and significantly increased that of the TA $(p<0.05)$. These results suggest that 30 minutes of PMS is effective in reducing motor neuron excitability of the TS in spastic hemiplegia, thus providing a safe and economical method for treating stroke patients ${ }^{[17]}$.

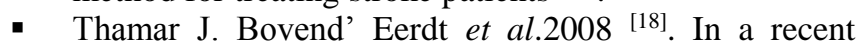
study done for the effectiveness of stretching to reduce spasticity, a systemic literature was performed using a database which included adults receiving stretching technique to reduce spasticity in which 6 of 10 studies reported statistically significant reduction in spasticity. The study investigated the general effect of stretching on spasticity and to explore the complexity of stretching in patients with spasticity. Two researchers independently performed a systematic literature search using the databases: Medline, PEDro, Cochrane library, Web of Science, CINAHL, and Allied and Complementary Medicine. Studies on adults receiving a stretching technique to reduce spasticity were included. study concluded that there is a wide diversity in studies investigating the effects of stretching on spasticity, and the available evidence on its clinical benefit is overall inconclusive. We recognize the need for consensus on a paradigm for stretching and for good-quality studies. Future research should address this issue and should investigate the clinical importance of the short- and long-term effects.

- Sandra L. Whisler, M.S., et al, 2011. [19] To assess effectiveness of myofascial release for 6 months of spasticity. Modified ashworth scale as an outcome measure was studied in six children with cerebral palsy. Myofascial release technique was given over a period of six months. Caregivers were taught these techniques and provided with an evaluation sheet to assess clinical and behavioral parameters during the study. The study concluded that the myofascial release therapy can be beneficial in decreasing muscle spasticity.

- Salvi Shah,et al 2012. ${ }^{[20]}$ Myofascial release (MFR) refers to the manual massage technique for stretching the fascia and releasing bonds between fascia and integuments, muscles, bones, with the goal of eliminating pain, increasing range of motion, and balancing the body. The fascia is manipulated, directly or indirectly, allowing the connective tissue fibres to reorganize themselves in to a more flexible, functional fashion. The purpose of the myofascial release is to release restrictions (barriers) within the deeper layers of fascia. This is accomplished by a stretching of the muscular elastic component of the fascia, along with the crosslink, and changing the viscosity of the ground substance of the fascia. Evidence shows that MFR is safe, effective and designated to be utilized with appropriate modalities, mobilization, exercise and flexibility programs, neurodevelopment treatment (NDT), sensory integration and movement therapy

\section{Discussion}

- In the present study, 30 male and female subjects with chronic stroke between the age of 35 to 75 years were selected according to inclusion and exclusion criteria. The subjects were divided into 3 groups: Group A, group B \& group C by random allocation by chit method.

- Group A received faradic electrical muscle stimulation for wrist extensors \& myofascial release for flexors of forearm.

- Group B received faradic muscle stimulation for wrist extensors and passive stretching for forearm and wrist flexors.

- Group $\mathrm{C}$ received faradic muscle stimulation for wrist extensors and conventional home exercise program in form of self-stretching of wrist and forearm flexors and active exercise of the hand.

- The pre and post outcome measure was modified sollerman hand function test. (MSHFT)

- In this study hand function was assessed using Modified Sollerman Hand Function Test to perceive the effectiveness of Myofascial release with faradic muscle stimulation and Passive stretching with faradic muscle stimulation in patients with chronic hemiplegic stroke.

- Male gender itself is stronger risk factor as stated in earlier studies. Studies have often mentioned the incidence rates to be about $25 \%$ to $30 \%$ higher among men than women, also review accounts for an overall risk of increase in poor recovery noted in various studies in $33 \%$ of male stroke patients. ${ }^{21}$ In the present study however, there was no difference see in gender as far as involvement and recovery was concerned.

- In a Retrospective study, the participants comprised of 84 males and females, first-time stroke patients, were assessed for total FIM improvement between normalweight, overweight, and obese patients. Results showed that, the relative improvement of FIM score was significantly higher in normal-weight patients than in overweight patients, and improvement in overweight patients was significantly higher than in obese ones. Therefore, study showed that, rehabilitation is statistically significantly less effective in overweight and, particularly, in obese patients (evaluated by BMI). [22] In the present study it is not consistent with the findings from the previous research work. i.e., the results from the present study showed the improvement of the hand function was not affected by weight of the participant.

- Earlier research in relation to the timing of the therapy for stroke patients stated that, it has to start as early as possible after the brain lesion and is a common clinical believe that stroke patients past a certain time window are unlikely to benefit from motor rehabilitation, ${ }^{23}$ but 
in a prospective research study stated that, motor rehabilitation of stroke patients remains efficacious even in the "chronic" stage, i.e. several months or years after the acute stroke [24]. The result of the present study showed participants in all groups showed significant improvement in all the groups from pre-training to posttraining, hence it is consistent with the other findings that the duration of illness did not affect the recovery in hand function.

- The myofascial release (or deep tissue work) method works through engaging the myofascial tissue restrictive barrier, the tissue is loaded with a constant force until tissue release occurs. Indirect myofascial release seeks for changes in the myofascial structures by stretching, elongation of fascia, or mobilizing adhesive tissues. It is given slowly through the layers of the fascia until the deep tissues are reached.

- Burris Duncan, et al. Children between the ages of 20 months and 12 years with moderate to severe spastic cerebral palsy were enrolled in a randomized control study. The effectiveness of osteopathy in the cranial field, myofascial release, or both versus acupuncture was assessed and was measured by several outcome measures which included Gross Motor Function Scale and modified ashworth scale .Each child was scheduled to be treated with OMT for 10 sessions of MFR over a 24-week period: weeks 1-4, once every week (4 treatments); weeks 5-8, once every other week (2 treatments); and weeks 9-24, once every month (4 treatments). Each treatment session lasted 30 to 60 minutes, there were 3 arms in the study, OMT (Osteopathy in Cranial Field, Myofascial release or both), Acupuncture and Control (Non-Therapeutic attention). The results concluded that a series of treatment using osteopathy in the cranial field, myofascial release or both improved motor function in children with moderate to severe cerebral palsy with initial 4 treatment sessions by marked decrease in spasticity ${ }^{[25]}$. These findings are consistent with the present study, i.e. myofascial release given with faradic electrical muscle stimulation showed greater improvement from pre-training to $14^{\text {th }}$ day of intervention in group A. Modified Sollerman Hand Function Test from pre-training to $14^{\text {th }}$ day of intervention showed significant improvement. The reason for improved hand function was because of reduction in spasticity and hence improved function.

- Osteopathic physicians have proposed that the greatest benefit of myofascial release for patients with cerebral palsy will be seen in children younger than 2 years of age and in children with limited neurologic involvement. Most of the children were at an age when the brain is no longer developing at a rapid pace and when plasticity is presumed to be reduced. Yet, from an osteopathic perspective, children with $\mathrm{CP}$, improved circulation with increased oxygenation and removal of waste products, result from myofascial release. ${ }^{26}$ These findings are consistent with the present study that the age of the participants did not affect the hand function in stroke patients ,hence myofascial release has been proven to be beneficial not only in children's but also in adult patients with mild to moderate spasticity. The possible mechanism for reduction in spasticity can be increased circulation of neurons in less plastic brain of adults could improve motor functions.

- Passive muscle stretching is a common physical therapy for decreasing the spasticity in stroke patients. It has been reported that prolonged passive muscle stretching improves the range of movements and reduces spasticity. Passive muscle stretching activates Golgi tendon organs and inhibits the excitability of alpha motor neurons. Reduced motoneuronal excitability leads to an increase in the extensibility of soft tissues. Current evidence supports the effectiveness of passive stretching in adults with chronic stroke.

- Ten stroke survivors with wrist spasticity and ten healthy control subjects received intervention of 60-min wrist stretching. Stiffness was evaluated pre- and postintervention. Compared to healthy controls, the wrist position of the stroke survivors was shifted towards extension. Stroke survivors showed significantly decrease in stiffness after the stretching intervention, especially in flexion. Wrist range of motion was also increased by stretching. [27] These findings were consistent with the present study, that passive stretching given for 30 minutes reduces spasticity from pretraining to post training. The reason for reduction in stiffness can be stretching significantly improved the force output of the impaired wrist and forearm muscles in stroke survivors. Also only passive stretching was given with no other treatment protocol, in the present study passive stretching was given along with the faradic electrical muscle stimulation and it showed significant improvement in function of hand from pretraining to post-training in group B.

- To investigate the passive and active changes of muscle-tendon units of plantar flexors induced by passive ankle stretching in stroke survivors, ten stroke survivors without any neurological disorder participated in the study. There were twelve intermittent stretching sessions of $5 \mathrm{~min}$ each (one hour in total). Post treatment, the active force-generating capacity of stroke survivors improved. [27] These findings are consistent with the present finding, i.e. there is significant improvement in the function of hand from pre-training to post training with passive stretching and faradic muscle stimulation.

- Faradic muscle stimulation increase blood flow to the area treated that make the area slight redness due to vasodilatation, also faradic current contracts the muscle like voluntary muscle contraction. To prevent fatigue the faradic, allow the muscle to relax after contraction.

- The faradic current increase metabolism and remove waste product and bring more blood supply and nutrients to muscle thus increase demand of oxygen and nutrients to muscle. Electrical stimulation will reeducate of muscle action. The electrical stimulation can help in minimize the extent of muscle atrophy.

- The influence of faradic electrical muscle simulation of the extensor and flexor carpi radialis muscles on bio mechanical and functional movement parameter was compared with the effect of standardized active repetitive exercises of the hand. Twelve patients suffering from ischemic lesions in the territory of MCA participated in the study. All the patients received electrical simulation for 20 minutes twice daily, in the third phase repetitive hand and finger training was 
conducted for $20 \mathrm{~min}$ daily. With the exception of spasticity, electrical simulation did not improve any bio mechanical or functional movement parameters. ${ }^{21}$ The present study demonstrated that electrical stimulation along with the active exercises for the hand given for 30 minutes once daily for 14 days improved hand muscle function from pre-training to post training in Group C .

- To evaluate constraint-induced movement therapy for chronic stroke patients, sixteen stroke patients (nine men and seven women; mean age 56.7 years; on average 28.9 months post stroke, five of whom were 69 months post stroke) were recruited. Outcome measures were Modified Motor Assessment Scale, Modified Sollerman Hand Function Test. After the training period, the mean motor performance improved significantly after two weeks of constraint-induced group therapy on Motor Assessment Scale and on Modified Sollerman Hand Function Test ${ }^{28}$ These findings were consistent with the present that the modified sollerman hand function test showed significant improvement in all the groups in 2 weeks intervention protocol to improve hand muscle function . MSHFT showed significant improvement in gross motor function than fine motor function.

- The pre and post treatment scores of MSHFT were statistically analyzed and compared. For, intragroup pre and post outcome measure values of MSHFT T-Test was used. For intergroup pre and post outcome measure values of MSHFT paired T- test was used.

- Mean values for MSHFT for Group A was: $4.2 \pm 0.15$; Group B was: $3.8 \pm 0.55$; Group C -was: $2.2 \pm 0.43$

- The results show that all three techniques Myofascial release with Faradic muscle stimulation, Passive stretching along with Faradic muscle stimulation, conventional exercise protocol, i.e. Faradic muscle stimulation and Home exercise program are highly effective in improving grip strength and hand function but as compared to conventional exercises Myofascial release and Passive stretching were more effective.

\section{Conclusion}

In the present study, according to the results it is proved that in both male and female subjects with clinical diagnosis of stroke having impaired hand function, when given
Myofascial release with Faradic muscle stimulation showed improvement in grip strength and hand muscle function. Similarly, subjects treated with Passive stretching along with Faradic muscle stimulation showed improvements in grip strength and hand muscle function. Subjects treated with conventional exercise protocol, i.e. Faradic muscle stimulation and Home exercise program also showed improvement in hand function and grip strength.

All the 3 groups showed improvements in grip strength and hand muscle function, but as compared to conventional exercises Myofascial release and Passive stretching were more effective.

\section{Statistical analysis}

Table 1: Showing Gender Distribution In Group A, Group B And Group C.

\begin{tabular}{|c|c|c|c|}
\hline \multirow{2}{*}{ Gender } & \multicolumn{3}{|c|}{ Groups } \\
\cline { 2 - 4 } & Group-A & Group-B & Group-C \\
\hline Female & $\mathbf{3}$ & $\mathbf{4}$ & $\mathbf{7}$ \\
\hline Male & $\mathbf{7}$ & $\mathbf{6}$ & $\mathbf{3}$ \\
\hline Total & 10 & 10 & 10 \\
\hline
\end{tabular}

Table 2: Handedness

\begin{tabular}{|c|c|c|c|}
\hline \multirow{2}{*}{ Handeness } & \multicolumn{3}{|c|}{ Groups } \\
\cline { 2 - 4 } & Group-A & Group-B & Group-C \\
\hline Right & 9 & 9 & 9 \\
\hline Left & 1 & 1 & 1 \\
\hline Total & 10 & 10 & 10 \\
\hline
\end{tabular}

Table 3: Affected Side

\begin{tabular}{|c|c|c|c|}
\hline \multirow{2}{*}{ Affectedside } & \multicolumn{3}{|c|}{ Groups } \\
\cline { 2 - 4 } & Group-A & Group-B & Group-C \\
\hline Right & 7 & 7 & 7 \\
\hline Left & 3 & 3 & 3 \\
\hline Total & 10 & 10 & 10 \\
\hline
\end{tabular}

Table 4: Graph showing MSHFT values of Group A

\begin{tabular}{|c|c|c|c|}
\hline & Pre & Day 7 & Post \\
\hline Mean & 35.2000 & 36.5000 & 39.4000 \\
\hline SD & 8.05260 & 7.96171 & 7.89092 \\
\hline P value & $<0.05$ & $<0.05$ & $<0.05$ \\
\hline
\end{tabular}

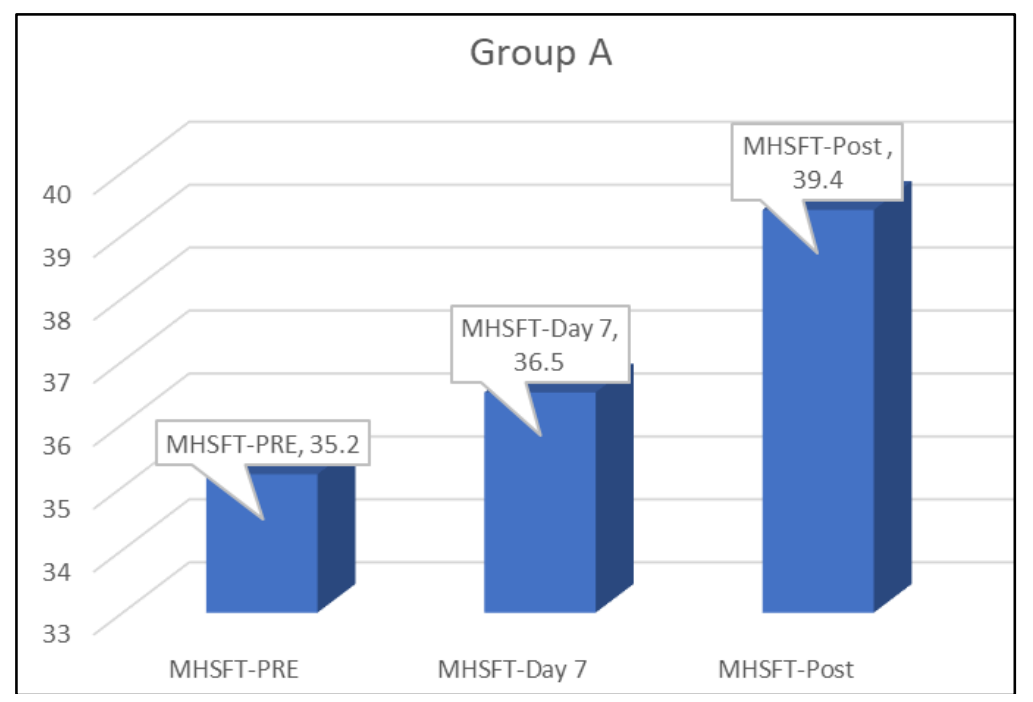

Group A: Showing Intra Group Comparision Of Mean Of Mshft In 
Table 5: Graph showing MSHFT values of Group B

\begin{tabular}{|c|c|c|c|}
\hline & Pre & Day 7 & Post \\
\hline Mean & 36.2000 & 37.2000 & 40.0000 \\
\hline SD & 11.52582 & 11.74545 & 12.11060 \\
\hline P value & $<0.05$ & $<0.05$ & $<0.05$ \\
\hline
\end{tabular}

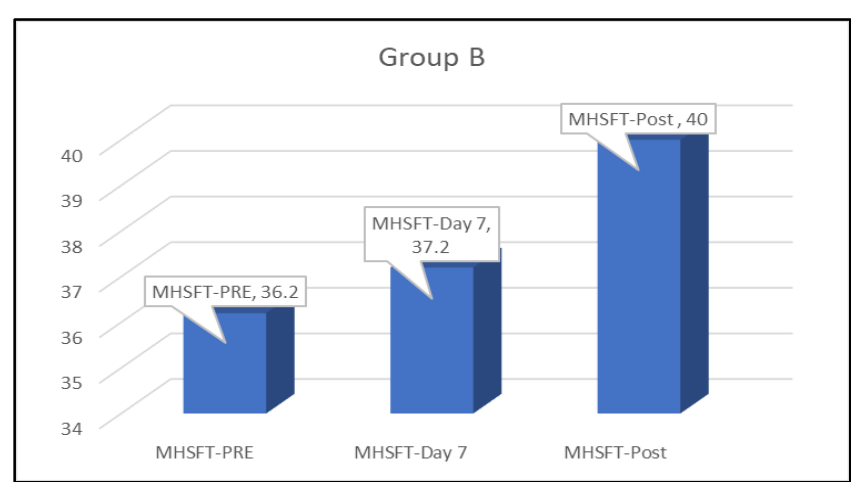

Group B: Showing Intra Group Comparision Of Mean Of Mshft In

Table 6: Graph showing MSHFT values of Group C

\begin{tabular}{|c|c|c|c|}
\hline & Pre & Day 7 & Post \\
\hline Mean & 36.5000 & 37.3000 & 38.7000 \\
\hline SD & 12.11289 & 11.778558 & 12.08351 \\
\hline P value & $>0.05$ & $<0.05$ & $<0.05$ \\
\hline
\end{tabular}

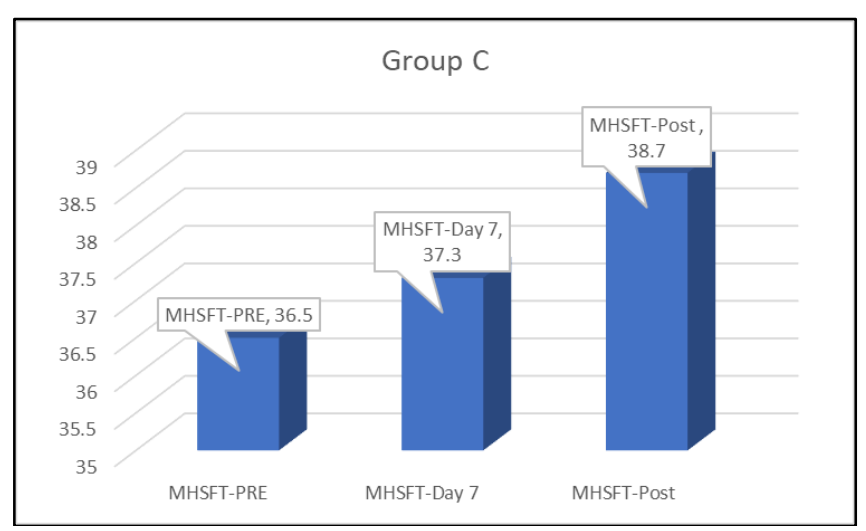

Group C: Showing Intra Group Comparision Of Mean Of Mshft In

Table 7: values of post treatment scores of MSHFT of group A group B \& group C

\begin{tabular}{|c|c|c|c|}
\hline & Group A & Group B & Group C \\
\hline Mean & 4.2000 & 3.8000 & 2.2000 \\
\hline Standard deviation & 7.89092 & 12.11060 & 12.08351 \\
\hline P value & $<0.05$ & $<0.05$ & $<0.05$ \\
\hline
\end{tabular}

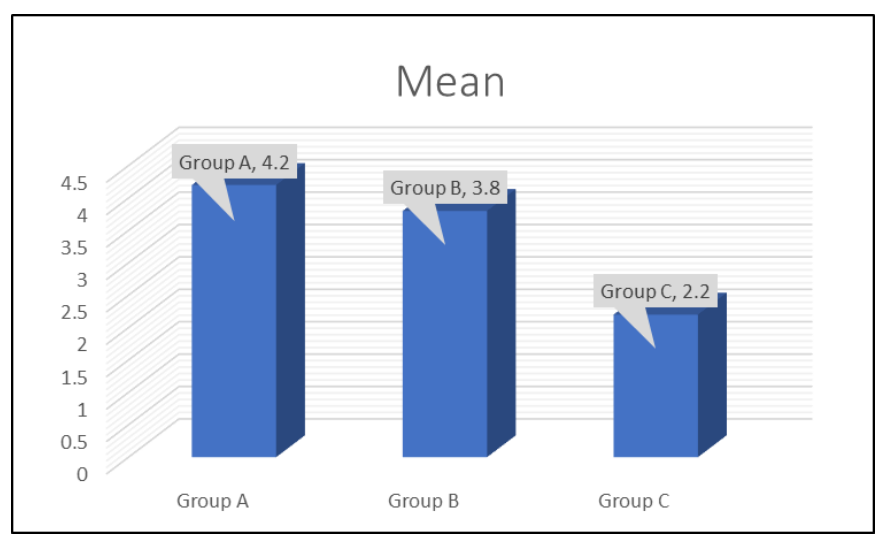

\section{Result}

The present study was done to see the effect of myofascial release with faradic electrical muscle stimulation and passive stretching with faradic electrical muscle stimulation to improve hand muscle function in chronic stroke patients.

The pre and post treatment scores of MSHFT were statistically analyzed and compared. For, intragroup pre and post outcome measure values of MSHFT T-Test was used. For intergroup pre and post outcome measure values of MSHFT paired T- test was used. Mean values for MSHFT for Group A was: $4.2 \pm 0.15$; Group B was: $3.8 \pm 0.55$; Group $\mathrm{C}$ was: $2.2 \pm 0.43$. The obtained $\mathrm{p}$ value was $<0.05$ (significant).

\section{Limitations}

- Study has small sample size

- Wrist range of motion was not taken into consideration.

\section{Future scope}

- Studies with larger duration could be taken with larger follow up period to assess the long-term benefits of the intervention.

- larger sample size could be taken into consideration.

- Range of motion should be taken into consideration

- Grading of spasticity could have been taken into consideration.

\section{References}

1. Sims NR, Muyderman H. Mitochondria, oxidative metabolism and cell death in stroke. Biochimica et Biophysica Acta (BBA)-Molecular Basis of Disease. 2010; 1802(1):80-91.

2. Ga D, Fisher M, Macleod M, Davis Sm. Stroke. Lancet. 2008; 371(9624):1612-23.

3. Deb P, Sharma S, Hassan KM. Pathophysiologic mechanisms of acute ischemic stroke: An overview with emphasis on therapeutic significance beyond thrombolysis. Pathophysiology. 2010; 17(3):197-218.

4. Feldman EL, Cornblath DR, Porter J, Dworkin R, Scherer S. National Institute of Neurological Disorders and Stroke (NINDS): Advances in understanding and treating neuropathy, 24-25 October 2006; Bethesda, Maryland. Journal of the Peripheral Nervous System. 2008; 13(1):1-6.

5. Hill MD. Diagnostic biomarkers for stroke: a stroke neurologist's perspective; Clinical Chemistry. 2005; 51(11):2001-2002.

6. Fisher CM. The arterial lesions underlying lacunes. Acta neuropathologica. 1969; 12(1):1-15.

7. Lange B, Flynn S, Rizzo A. Initial usability assessment of off-the-shelf video game consoles for clinical gamebased motor rehabilitation. Physical Therapy Reviews. 2009; 14(5):355-363.

8. Lange B, Flynn S, Rizzo A. Initial usability assessment of off-the-shelf video game consoles for clinical gamebased motor rehabilitation. Physical Therapy Reviews. 2009; 14(5):355-363.

9. Woldag H, Hummelsheim H. Evidence-based physiotherapeutic concepts for improving arm and hand function in stroke patients. Journal of neurology. 2002; 249(5):518-528.

10. Mann GE, Burridge JH, Malone LJ, Strike PW. A pilot study to investigate the effects of electrical stimulation on recovery of hand function and sensation in subacute 
stroke patients. Neuromodulation: Technology at the Neural Interface. 2005; 8(3):193-202.

11. Petkunas Byrne D, Ridgeway EM. Considering the whole body in treatment of the hemiplegic upper extremity. Topics in Stroke Rehabilitation. 1998; 4(4):14-34.

12. American Osteopathic Association. Foundations of osteopathic medicine. Lippincott Williams \& Wilkins. Edn 2010; 3:700-722.

13. DiGiovanna EL, Schiowitz S, Dowling DJ. (Eds.). An osteopathic approach to diagnosis and treatment. Lippincott Williams \& Wilkins. EDN 2005; 3:442-452.

14. Appleton B. Stretching, and flexibility: types of stretching. Sports Medicine: Chicago. version 2002; $1: 36$

15. Joanna Powell MCSP, Pandyan AD, Granat M, Margaret Cameron MCSP, Stott DJ. Electrical Stimulation of Wrist Extensors in Poststroke Hemiplegia. Stroke. 1999; 30:1384-1389.

16. Amit Bakhtiary, Elham Fatemy. Does electrical stimulation reduce spasticity after stroke? A Randomized Controlled Study. Clinical Rehabilitation 2008; 22(5).

17. Tsai KH, Yeh CY, Chang HY, Chen JJ. Effects of a single session of prolonged muscle stretch on spastic muscle of stroke patients. Proceedings-National Science Council Republic of China Part B Life Sciences. 2001; 25(2):76-81.

18. Bovend'Eerdt TJ, Newman M, Barker K, Dawes H, Minelli C, Wade DT. The effects of stretching in spasticity: a systematic review. Archives of physical medicine and rehabilitation. 2008; 89(7):1395-1406.

19. Whisler SL, Armstrong M, Vickers J, Associates M. Effects Of Myofascial Release On Children With Cerebral Palsy: Six Case Reports. 2012; 8(3):199-205

20. Akta Bhalara. International Journal of Health Sciences and Research, Myofascial Release Salvi Shah1. 2012; 2(2):69-77

21. Lin HC, Lin YJ, Liu TC, Chen CS, Chiu WT. Urbanization and stroke prevalence in Taiwan: analysis of a nationwide survey. Journal of urban health. 2007; 84(4):604-614.

22. Kalichman L, Rodrigues B, Gurvich D, Israelov Z, Spivak E. Impact of patient's weight on stroke rehabilitation results. American journal of physical medicine \& rehabilitation. 2007; 86(8):650-655.

23. Jørgensen HS, Nakayama H, Raaschou HO, ViveLarsen J, Støier M, Olsen TS. Outcome and time course of recovery in stroke. Part I: Outcome. The Copenhagen Stroke Study. Archives of physical medicine and rehabilitation. 1995; 76(5):399-405.

24. Whitall J, Waller SM, Silver KH, Macko RF. Repetitive bilateral arm training with rhythmic auditory cueing improves motor function in chronic hemiparetic stroke. Stroke. 2000; 31(10):2390-2395.

25. Shojania KG, Duncan BW, McDonald KM, Wachter RM, Markowitz AJ. Making health care safer: a critical analysis of patient safety practices. Evid Rep Technol Assess (Summ). 2001; 43(1):668.

26. Frymann V, Carney R, Springall P. Effect of osteopathic medical management on neurologic development in children. J Am Osteopath Assoc. 1992; 92(6):729-44.
27. Gao F, Ren Y, Roth EJ, Harvey R, Zhang LQ. Effects of repeated ankle stretching on calf muscle-tendon and ankle biomechanical properties in stroke survivors. Clinical biomechanics. 2011; 26(5):516-522.

28. Brogårdh $\mathrm{C}$, Sjölund $\mathrm{BH}$. Constraint-induced movement therapy in patients with stroke: a pilot study on effects of small group training and of extended mitt use. Clinical rehabilitation, 2006; 20(3):218-227. 